\title{
Reflexiones elementales sobre educación y contabilidad
}

\author{
Rosa Isabel González Muñoz ${ }^{1}$
}

\begin{abstract}
Resumen
El documento propone una reflexión sobre las dinámicas de la educación en el campo de la contabilidad, haciendo énfasis en los problemas elementales de la enseñanza (Qué, Cómo y para qué enseñamos) y como estas preguntas se resuelven en el campo específico de la contabilidad, la autora cuestiona las formas tradicionales de transmisión de conocimientos, por su falta de actualización y sentido transdisciplinar.
\end{abstract}

La autora usa el concepto de competencia para identificar el conjunto de competencias que los contadores del mundo contemporáneo requieren, plantea la necesidad de que los programas de formación profesional evolucionen más allá del saber hacer, y proporcionen nuevas herramientas y capacidades a los futuros profesionales.

1 Contadora pública de la Universidad Nacional de Colombia, MBA de la Universidad de los Andes, candidata al Doctorado en Administración de la Universidad de Tulane en los Estados Unidos. Profesora asistente en la Facultad de Administración de la Universidad de los Andes desde junio de 2003. Actualmente se desempeña como directora del Programa del pregrado en Contaduría Internacional de la Universidad de los Andes. Citar como: González, R.I. (2017). Reflexiones elementales sobre educación y contabilidad. Activos, 15(28), 35-46. DOI: https://doi.org/10.15332/s0124-5805.2017.0028.02 


\title{
Palabras Claves
}

Educación contable; Formación por Competencias; Globalización; Capacidades Profesionales; Docencia

\section{Elementary reflections on accounting and education}

\begin{abstract}
The document proposes a reflection about the dynamics of education in the field of accounting, emphasizing in the elementary problems of teaching (What, How and for what we teach) and how these questions are solved in the specific field of accounting, the author questions the traditional ways of transmitting knowledge, for its lack of updating and transdisciplinary sense.
\end{abstract}

The author uses the concept of "training by competences" to identify the set of competences that the accountants of the contemporary world require, raises the need for professional education programs to evolve beyond knowhow, and provide new tools and skills to future professionals.

\section{Keywords}

Accounting Education; Training By Competences; Globalization; Professional Capabilities; Teaching 
En primer lugar, quiero agradecer a la Universidad Santo Tomás por esta invitación. Realmente este es un espacio muy interesante para poder compartir con ustedes algunas ideas de lo que uno piensa como profesor; creo que, en ese sentido, pensar y tratar de encaminar esos pensamientos son parte de nuestro día a día.

Comienzo diciendo que realmente este es un espacio para reflexionar acerca de nuestro día a día como profesores y básicamente ir evolucionando en torno a tres preguntas que yo me hice cuando me hicieron la invitación: ¿qué enseñamos? (esta sería una pregunta básica), ¿cómo enseñamos? y, finalmente, ¿para qué enseñamos? Estas dos últimas preguntas son un poco más filosóficas. A continuación, lo que voy a hacer es compartirles mis propias reflexiones alrededor de esos tres interrogantes.

Para iniciar, quiero retomar un tema que se presentaba anteriormente, y es el tema de las competencias. Hay un texto muy bonito que desarrolló el profesor Luis Enrique Orozco (Orozco, 2016) en la Universidad de los Andes y que se titula Enfoque de competencias en materia de estructuras curriculares, que no solamente está pensado para el tema de contabilidad como campo disciplinario específico, sino que en realidad es una reflexión global de lo que significan las competencias en educación superior. Básicamente, lo que Orozco dice es que debemos ser muy conscientes de que el concepto de competencia no necesariamente es un concepto académico, sino que en realidad es un concepto que tomamos prestado del mundo laboral. Todo lo que hacemos en competencias es tratar de generar espacios donde copiamos lo que pasa en el mundo laboral, eso es una competencia.

Más adelante en su texto habla de los componentes de la competencia y señala los siguientes tres componentes:

- La comprensión de unos fundamentos conceptuales. Para ser competente, yo debo tener un campo disciplinario, pero cuando se está hablando de educación superior, es necesario indicar hasta dónde debería llegar esa comprensión, por eso es importante indicar cuáles son los fundamentos disciplinarios en cualquier área de formación. 
Hay unos análisis muy fuertes que indican el problema de analizar las disciplinas como silos. Entonces, en este lado está la contabilidad, por allá arriba está la administración, por el otro lado está la economía, y realmente vivimos en un ambiente en el que el enfoque interdisciplinario es necesario para comprender qué está detrás de los procesos. Creo que eso nos ayuda mucho a que nos dediquemos a la discusión sobre qué normas debemos aplicar; pero no es solo un tema de normas, es de cuáles son los fundamentos conceptuales que las definen. Si entendemos los fundamentos conceptuales, ante cualquier marco conductual, cualquier marco normativo, debemos estar en capacidad de comprenderlo, porque comprendemos lo que está detrás.

- El desarrollo de habilidades. En este tema es clave desarrollar habilidades en el sentido de qué sé hacer. El saber hacer algo es muy importante en cualquier disciplina, pero en la contabilidad, en los temas contables y de la profesión contable se vuelve un tema vital. Como todos aquí en este auditorio (o casi todos) son contadores, entonces yo voy a permitirme decir algo polémico: a nosotros nos formaron como contadores, y creo que nos quedó una sensación de que uno estaba detrás de ese gran escenario donde se tomaban las decisiones. De hecho, mucha gente dice: "Es que usted prepara la información para que, por favor, se la pase a otros donde toman las decisiones".

Yo, particularmente, siento que en el tema de cómo nosotros participamos en los procesos de toma de decisiones no se trata de conocimiento, no es que no sepamos ni conozcamos, es más bien un problema de habilidad para comunicarnos, habilidad para trabajar en equipo, habilidad para poder entender qué es ejercer liderazgo, ejercerlo eficazmente y saber que uno también es capaz de dar el paso al frente.

Digamos que en mi proceso de formación como contadora me parecía impresionante que nosotros hablábamos en forma de código morse: los contadores ya no hablaban, había que decirles "la 141727", y nos entendíamos, pero eso hacía que nadie más nos entendiera; era muy bonito entre nosotros, pero nadie más nos entendía. Yo creo que ahí hay un trabajo muy 
importante en el desarrollo de habilidades. Por último, hay un tema que a mí me llama mucho la atención, porque es un gran reto: es el temple y la templanza de la actitud frente al mundo y a su disciplina.

Es un poco en el sentido de lo que expresaba la profesora Alejandra Patiño (Patiño, 2017): no se trata de lo que usted sabe hacer, sino de que usted tenga el temple y la templanza, de tener una posición y sustentarla. Entonces, en la formación contable también viene algo que no solamente se enmarca en lo que pasa en estos salones, sino en ese proceso de formación continua, de cómo la actitud del contador también habla, de cómo yo veo la profesión y la defiendo: profesionales críticos, no criticones, con una visión global para entender qué pasa en lo local.

A mí me sorprende un hecho sobre el que he venido reflexionando recientemente: el grado de "parroquialismo" con el que nos comportamos los contadores aquí en Colombia. En la reunión de los cien años de la Asociación Americana de Contadores, en agosto del año pasado, una profesora norteamericana hizo una intervención en defensa del nacionalismo contable, un tema que aquí resulta desgastado y hasta pasado de moda. Yo creo que el nacionalismo contable no es un tema pasado de moda, sino que en realidad hay una preocupación por entender lo local; sin embargo, para ello también hay que tener sensibilidad respecto a qué son las tendencias globales, y muchos discursos aquí del nacionalismo contable quieren devolvernos al siglo $\mathrm{XX}$, y en eso consiste ese parroquialismo. Es necesario no descalificar ni alinearnos con una idea directamente sin un proceso de análisis que nos permita entender la realidad del mundo, por ello, creo que el debate nacionalismo vs. globalización es, en sí mismo, un debate parroquial que no tiene ningún sentido.

- Sensibilidad a otros enfoques y a otras disciplinas. A veces nosotros, los contadores, somos percibidos por las demás personas como soldados de los números, personas que perdimos sentido de sensibilidad, que trabajamos en organizaciones donde perdemos sentido de lo que nosotros hacemos. Estas apreciaciones tienen unos impactos mucho más amplios que lo simplemente anecdótico. La sensibilidad 
y ese formar una actitud, un temple y una templanza son parte de la responsabilidad de la formación de los contadores, y eso se logra cuando la contabilidad se comprende no solo en sí misma, sino también en su interrelación con otros campos disciplinarios.

Estos tres elementos son fundamentales en el modelo que Orozco (2016) presenta y que viene como parte de las tendencias de la educación superior a nivel mundial. Además de eso, es necesario desarrollar capacidades de discernimiento ético, porque no se trata de ser buenos y de portarnos bien, se trata de que usted es responsable desde su ámbito profesional. Entonces, ese discernimiento ético es parte de tener conocimiento, de tener habilidades y además la actitud y la templanza para poder defenderlas.

Creo que ese es un elemento que proponen las tendencias globales que comparto plenamente, es un elemento que se debe tener muy presente en lo que hacemos en nuestro día a día. Ahí aparece la competencia, cuando todos esos elementos logran encajar y construir una forma de enfrentar un proceso de formación.

Para mí, el debate sobre competencias plantea una serie de oportunidades que no son pequeñas, porque implican un cambio importante de paradigma. Implica una ruptura con el paradigma de la educación sumativa - que, creo, todos nosotros lo vivimos, en el cual hay que aprender muchas cosas, hay que saber todo antes del día del grado- y el paso a un paradigma de la educación formativa, con cuidado, por supuesto.

Porque el hecho de que hayamos tomado prestado el concepto de competencia del mundo laboral no nos puede hacer perder de vista que nosotros somos universidades, y las universidades tenemos una responsabilidad social, más allá de entrenar personas que van a insertarse en el mundo laboral: tenemos responsabilidades investigativas, tenemos responsabilidades en muchos ámbitos que debemos saber equilibrar. Entonces, creo que la respuesta al "qué se enseña" es que estamos enseñando mucho más que normas o estándares de contabilidad, estamos enseñando mucho más que leyes. Más allá de estar enseñando, estamos formando. 
Luego planteé la pregunta sobre el cómo se enseña. Todos hemos tenido experiencias acerca de cómo le enseñan a uno, y creo que ese cambio en el paradigma también transforma la forma de las que podrían ser metodologías de enseñanza y nos da un ambiente bien complejo respecto a una educación en la que la universidad de pregrado no es todo el proceso de formación, sino que es una parte de un proceso más grande. Y yo creo que eso, como académicos, nos debe ayudar a tranquilizarnos un poco en el sentido de que antes uno decía "no, es que en diez semestres hay que enseñarles todo, porque lo que no aprendieron para el día del grado nunca más nadie les va a decir nada", y resulta que el punto es que, con todos los cambios, con la complejidad de lo que enfrentamos, pues no vamos a poder enseñarles todo.

Entonces, ¿qué es lo que deberíamos generar como ambiente en nuestros salones de clase? Que nuestros estudiantes aprendan a aprender, que tengan claros los elementos conceptuales fundamentales, que tengan muy buenas habilidades que les permitan a ellos saber y entender que es natural que a mí me cambien las normas, que a mí me cambien las reglas y que yo pueda entender por qué, y si las cambiaron tanto, saber qué fue lo que cambiaron.

El tema de las metodologías debe llevar a enseñar a aprender más que a enseñar temas. En ese mismo enfoque de largo plazo está el tema de ser conscientes de que lo que nosotros hacemos tiene límites, porque nosotros, como les decía al tomar esos conceptos de competencias del mundo laboral y trasladarlos a un mundo académico, no tenemos todos los elementos. Entonces, hay que ser consecuentes. La competencia, vista como un todo, realmente necesita del desempeño profesional porque se nutre de la experiencia, y eso, por más esfuerzos que hagamos con todas las metodologías que pudiéramos trabajar, es muy difícil traerlo literalmente al salón de clase; de manera que yo creo que hay que ser cuidadosos.

Además, respecto a nuestros campos disciplinarios, uno siente que cada vez son más complejos. No sé cómo sería hace unos años, pero cada vez uno ve cada uno de los campos disciplinarios en una evolución permanente, cada vez más complejos, que hacen que uno se pregunte si un 
programa del nivel de pregrado puede garantizar que el estudiante tenga una formación en profundidad en todas las áreas o si mejor se debe pensar en una formación que le abra los campos con una perspectiva amplia y que posteriormente venga una especialización, que nazca de su propio interés, en la cual complemente su conocimiento.

Yo recuerdo que, cuando estaba estudiando, el tema de auditoría a mí me parecía como un mundo de "esto no es lo mío". Entonces, si realmente un estudiante no tiene potencial para desarrollarse en ese campo, obligarlo a especializarse en esa área no tiene sentido; además, probablemente cuando salga a su mundo laboral no le va a encontrar el sentido. Creo que hay que ser cuidadosos también porque podemos llegar hasta un punto, pero también ellos van a evolucionar y tendrán que decidir cómo armarán su perfil de formación.

Nuestro rol como profesores tiene que cambiar, y aquí quiero decir una cosa que podría sonar a herejía en este auditorio: si la educación superior parte de la idea de que lo importante es que los profesores sepan mucho, eso no les aporta mucho a los estudiantes si ese enfoque no se transforma en uno en el que el docente se constituya en un activo importante para el proceso de formación de sus estudiantes, lo cual implica que seguramente no vamos a tener todas las respuestas correctas en nuestra labor como profesores, sino que, más bien, vamos a confrontar a nuestros estudiantes con las preguntas adecuadas.

Yo no sé si ustedes han tenido esa sensación en el salón de clase de que uno llega y hay muchos pares de ojos mirándolo a uno, como diciendo "por favor, profesor, diga usted la última palabra, por favor, por Dios, dígame", y uno cae muy fácilmente en la tentación, porque como profesor quiere entregar lo que uno considera que es lo más adecuado para que ellos comprendan; les facilitamos el proceso, pero también los marginamos de un proceso que es de ellos y es el de hacerse preguntas.

Hacernos preguntas nos va educando en el proceso de aprender. Aquí quiero compartirles unas charlas, de esas charlas bonitas de pasillo que 
tuve con el profesor Carlos Dávila. El profesor Dávila decía que aprender es doloroso. Tal vez ustedes conocen un artículo del profesor Dávila titulado "Si nuestros estudiantes son clientes", en ese documento se indica que no puede ser que nuestros estudiantes sean clientes, porque cuando mi estudiante es un cliente, lo que hago es tratarlo como tal y mi objetivo es hacerle la vida súper amable. Pero resulta que el proceso de formación no es un proceso amable, es un proceso doloroso porque precisamente debe confrontar a esa persona con muchas preguntas que dejan un sinsabor, pero que usted debe orientar para que ese sinsabor rápidamente sea puesto a favor del proceso formativo.

Todo lo anterior nos lleva a discutir sobre las metodologías empleadas en el salón de clase, y creo que en eso somos actualmente sujetos de todo el marco de las metodologías activas, que es una tendencia global en la que todo tiene que estar centrado en el participante, y yo creo que en esto también hay una oportunidad muy interesante. Vale la pena pensar sobre cómo uno activa a los estudiantes, y eso para docentes como nosotros es difícil, porque nos implica tener que ceder el protagonismo en el aula, lo cual es difícil.

Así que cuando a uno le dicen "no, mire, es que hay un caso Harvard que habla de una empresa que está en Estados Unidos, que tiene toda la información y que es perfecta y tiene este problema”, entonces a veces los estudiantes dicen "pues chévere, pero eso está como lejos". Entonces aquí creo que hay una labor interesante: dado que nosotros, como comunidad académica, desarrollamos investigación puesta al servicio de nuestros estudiantes, que les permita a ellos moverse entre lo local y lo global de manera que ellos puedan tener un contexto de qué es lo que está sucediendo, la labor de investigación nos permite tener evidencia, tener información de primera mano que nutra lo que sucede en el salón de clase.

En el contexto de la Universidad de los Andes tenemos la fortuna de contar con un gran número de profesores con doctorado, y el esfuerzo permanente es que esas investigaciones que hacemos en la Universidad no solo sirvan para llenar los anales y las revistas y tener puntajes, sino que 
eso esté en el salón de clase, y eso exige un proceso de pertenencia con las temáticas y un vínculo entre la formación y la investigación.

Yo creo que en el campo contable también tenemos todo el espacio por explorar en ese tipo de oportunidades de investigación en diferentes niveles (investigación científica e investigación aplicada) y de abordar todas las tendencias de producción intelectual, las cuales también ayudan a que las experiencias de conocimiento de nuestros estudiantes sean muchísimo más enriquecidas.

Por último, quiero apuntar a tratar de responder la pregunta ¿para qué enseñamos? No sé si todos saben que la Asociación Americana de Contadores (American Accounting Association, 2017) tiene un modelo de enseñanza que comienza por definir qué es la contabilidad. Ese modelo me parece muy interesante porque, según él, la contabilidad arranca de la comprensión de un entorno y una realidad de una organización, aplica lo que llaman el juicio profesional o el criterio profesional contable para la producción de información útil para la toma de decisiones.

¿Todo esto para qué? El modelo dominante aquí en Colombia seguramente va a responder que es para que alguien genere mucho dinero, para que una organización genere utilidades. El modelo de la Asociación Americana de Contadores dice que no, que el modelo contable es para generar prosperidad en la sociedad. Uno puede estar más cerca o menos cerca de estos modelos, pero creo que el mensaje es muy potente: nosotros enseñamos para formar unos profesionales que tengan la versatilidad de entender que, más allá del débito y el crédito, que más allá de la declaración de renta y los papeles de auditoría, lo que nosotros hacemos en el día a día es contribuir a la prosperidad de la sociedad.

Lo usual es que los contadores se pierdan en esta definición, porque uno en su día a día tiene otras preocupaciones, pero creo que, si el mensaje es potente a nivel de la formación de pregrado, de pronto no hoy, no mañana, pero quizá en algunos años entendamos que la profesión contable tiene un rol vital para la sociedad, más allá de simplemente tener unos datos. Y en 
ese sentido creo que todos hemos leído esas palabras de Peter Baker: "Los contadores vamos a salvar el mundo", y él lo dijo en una instancia muy importante, un foro mundial que hablaba sobre la sostenibilidad, donde todo el mundo se rio de él. ¿Los contadores salvando el mundo? ¿Por qué?

Yo creo que el profesor Baker (2014) entiende que el contador tiene una relación grande con la visión de largo plazo de la sociedad, que ayudamos a entender cómo se genera no solamente la riqueza, sino cómo se generan otros elementos, como el componente social y el ambiental, cómo las empresas y las organizaciones trabajan en ese sentido. ¡Por supuesto que vamos a salvar el mundo si todos nos formamos! No solo nuestros estudiantes, sino todos nosotros nos formamos como profesionales de la contabilidad, con sólida fundamentación conceptual. Saber el para qué y el por qué se es contador y tener la disposición de hacerse preguntas constantemente. Desarrollamos las habilidades y las actitudes para interactuar en las organizaciones, somos responsables y mantenemos un comportamiento ético, eso nos va a fortalecer en nuestro criterio profesional y nos permite insertarnos en una sociedad del conocimiento. Además, en últimas, es un contador que entiende que hay que seguir aprendiendo, $y$ creo que nosotros también necesitamos entender y vivir eso, es un proceso natural entender que esto tiene un fin que es muchísimo mayor.

Si me preguntan si esto es fácil o difícil, pues es realmente como todo lo que vale la pena, no es fácil, no nos va a tomar cinco años, no nos va a tomar diez años, nos va a tomar muchísimo más tiempo, y creo que los resultados podemos verlos. Es entender que no estamos cambiando un sistema ni unas normas, sino que estamos cambiándonos a nosotros mismos. Yo soy muy optimista por naturaleza, pienso positivo, yo creo que nosotros hemos avanzado. De pronto a veces nos damos eso que coloquialmente llamamos látigo, jsí!, siempre pensamos en nuestras carencias: "Es que no tengo, es que los recursos, es que me falta, es que no hacemos", pero también creo que vamos por un camino en donde lo vamos a poder lograr si nos comprometemos en ese sentido y en cada una de las piezas. Eso fue lo que pensé cuando pensé en ustedes y en mí misma desde la profesión. Muchísimas gracias. 


\section{Referencias}

American Accounting Association: http://aaahq.org/Research/Books

Baker, C. (2014). The role of the state in corporate governance. Accounting History, 19, 291-307.

Orozco, L. E. (2016). Enfoque de competencias en materia de estructuras curriculares. Bogotá: Universidad de los Andes.

Patiño, A. (2017). Una revisión del concepto de competencia educativa. Revista Activos. 